(Versión electronica)

DOI: http://dx.doi.org/10.21704/ac.v78i2.1054

(C) Universidad Nacional Agraria La Molina, Lima - Perú

\title{
Estimación de la Huella Hídrica y de Carbono en los cultivos comunitarios de Quínoa (Chenopodium quinoa wild) correspondientes a la zona central de los andes ecuatorianos
}

\author{
Estimation of the Water and Carbon Footprint in the community crops of Quinoa (Chenopodium \\ quinoa wild) corresponding to the central zone of the Ecuadorian Andes
}

\author{
Lenin Javier Ramírez-Cando ${ }^{1 *}$; Richard Jachson Vilches Moreno ${ }^{1}$; Ronnie Xavier Lizano \\ Acevedo $^{1}$; Ingrid Estefanía Pinzón Colmenares ${ }^{1}$;Erika Lizbeth Sandoval Mena ${ }^{1}$ \\ *Autor de correspondencia
}

\begin{abstract}
Resumen
La Quinoa es un pseudo-cereal considerado estratégico para la Soberanía Alimentaria, por su alto valor nutritivo, por contener todos los aminoácidos esenciales y bajo contenido de gluten. Asimismo, en Ecuador se busca que los productos agrícolas andinos sean revalorizados y ecológicamente sostenibles. El objetivo principal que se planteó el estudio fue la estimación de dos indicadores de Impacto Ambiental, Huella Hídrica y Huella de Carbono, generados por el cultivo de Quinoa en los cantones de Cayambe-Pichincha-Ecuador comunidad Pucará y Colta-Chimborazo-Ecuador comunidad Mjipamba. Se recopiló información de inventario; desde la preparación del suelo hasta la gestión de residuos agrícolas; mediante entrevistas, análisis físico-químicos de muestras de suelo-fertilizantes y recolección de datos meteorológicos. Para la estimación de Huella de Carbono se siguió las directrices del IPCC. Para la estimación de la Huella Hidrica se aplicó la metodológica del Manual de Evaluación de Water Footprint Network y el procedimiento de cálculo del Manual de Evaporación de cultivo de la FAO, los datos fueron procesados en el programa CROPWAT 8.0. Los resultados reportaron una Huella hídrica de 356,49 y 98,49 $\mathrm{m}^{3} / \mathrm{t}$ en Pucara y Mijipamba respectivamente. La Huella de Carbono para esta actividad fue de 425,38 $\mathrm{kgCO}_{2}$ eq/ha en Mijipamba y 468,56 $\mathrm{kgCO}_{2}$ eq/ha en Pucara. La importancia de este tipo de estimación radica en contribuir con un desarrollo sostenible e impulsar al eco-etiquetado de productos andinos ligados a la certificación orgánica.
\end{abstract}

Palabras claves: Huella de Carbono; Huella Hídrica; cambio climático; Quinoa.

\begin{abstract}
Quinoa is a pseudo-cereal considered strategic for Food Sovereignty, because of its high nutritional value, containing all essential amino acids and low gluten content. Likewise, in Ecuador, Andean agricultural products are to be revalued and ecologically sustainable. The aim of the study was to assess two indicators of Environmental Impact, Water Footprint and Carbon Footprint, generated by the cultivation of Quinoa in Cayambe-Pichincha-Ecuador community Pucará and ColtaChimborazo-Ecuador community Mjipamba. Inventory information was collected; From soil preparation to management of agricultural residues; Through interviews, physical-chemical analyzes of soil-fertilizer samples and the collection of meteorological data. For Carbon Footprint estimation, the IPCC guidelines were followed. For estimation of the Hydraulic Footprint, the methodology of the Manual of Evaluation of the Water Footprint Network and the procedure of calculation of the Manual of Evaporation of cultivation of the FAO was applied, data was processed with CROPWAT 8.0 software. The results have shown water footprint of 356.49 and $98.49 \mathrm{~m} 3 / \mathrm{t}$ in Pucara and Mijipamba respectively and Carbon Footprint of $425.38 \mathrm{kgCO} 2 \mathrm{eq} / \mathrm{ha}$ in Mijipamba and $468.56 \mathrm{kgCO} 2 \mathrm{eq} / \mathrm{ha}$ in Pucara. The importance of this type of estimation lies in contributing to sustainable development and promoting eco-labeling of Andean products linked to organic certification.
\end{abstract}

Keywords: Quinoa; LCA; Carbo Footprint; water Footprint.

\section{Introducción}

En los últimos años se ha estado impulsando la producción de Quinoa (Chenopodium quinoa) un pseudocereal considerado estratégico para la soberanía alimentaria, esto se debe a las propiedades nutritivas, dietéticas, diversidad genética y a la capacidad de adaptación a diferentes ecotipos agroecológicos (FAO, 2017). El alto valor nutritivo de la Quinoa esta atribuido a su contenido 
proteico (10,4 a 17\% de proteína) (FAO, 2013; Jancurová, Minarovičová, \& Dandár, 2009). Por lo que varios autores la consideran como el "único alimento de origen vegetal que posee todos los aminoácidos esenciales"(Quiroga et al., 2014). Además, contiene ácidos grasos poliinsaturados, principalmente el $\omega 6$ (linoleico) seguido por el $\omega 9$ (oleico) y $\omega 3$ (ácido linolénico), a su vez contiene otras propiedades nutricionales como carbohidratos, vitaminas y minerales (Jancurová et al., 2009; PROINPA, 2011). Hay que mencionar, que a la Quinoa se le atribuye un alto aprecio comercial por su mínimo contenido de gluten, por lo que se le considera un alimento apto para pacientes con enfermedad celíaca (Zevallos, Herencia \& Ciclitira, 2014). Esto lleva a la inquietud respecto de la sostenibilidad de la Quinoa en Ecuador.

Los principales países productores "tradicionales" de Quinoa son: Bolivia, Perú y Ecuador, se estima que el $80 \%$ de la producción a nivel mundial está centrado en estos países (FAO \& ALADI, 2014), aunque en los últimos años se encuentras cultivos en la zona del mediterráneo. En Ecuador, la Quinoa se distribuye en las provincias de Pichincha, Imbabura, Carchi, Cañar, Cotopaxi, Chimborazo, Azuay y Tungurahua, donde el mayor rendimiento para el año 2016 se dio en Pichincha con 1,79 $\mathrm{t} /$ ha y la zona de menor productividad fue Tungurahua con 1,19 t/ha (Monteros, 2016). Siendo por esto un potencial cultivo no tradicional a fomentar, con el fin de dar alternativas a las comunidades andinas ecuatorianas.

La agricultura, es un sector que contribuye con el cambio climático, debido a las emisiones de Gases de Efecto Invernadero (GEI). Ecuador reportó que la agricultura para el año 2010, contribuyó con el 18,03 \% de las emisiones, considerándola el tercer sector generador de GEI, siendo el sector de Energía el que emitió principalmente con 44,49 $\%$ de las emisiones de GEI. En cuanto los suelos agrícolas, contribuyeron con $6795,00 \mathrm{Gg}$ de $\mathrm{CO}_{2}$-eq que corresponde el $46,81 \%$ dentro del sector agrícola, el mismo que emitió 14 515,94 Gg CO-eq.(MAE, 2016). Otra problemática asociada a la agricultura es el uso de recursos hídricos, ya que utiliza entre 2000 a 5000 litros/día/persona (Molden $\&$ de Fraiture, 2004). Según la Secretaria del Agua (SENAGUA), Ecuador utilizó el $76 \%$ del caudal para riego en el Sector Agrícola (Pérez Arcos, 2012).

Considerando lo anterior, se planteó como objetivo; estimar dos indicadores de impactos ambiental (la Huella de Carbono (HC) y la Huella Hídrica $(\mathrm{HH})$ ), en donde la $\mathrm{HC}$ se define como un "indicador de la cantidad de gases de efecto invernadero (GEI) generados y emitidos por una empresa o durante el ciclo de vida de un producto a lo largo de la cadena de producción" (CEPAL, 2012). Teniendo en cuenta que los GEI de mayor preocupación para el Sector de Agricultura, Silvicultura y Otros Usos de la Tierra denominado sector AFOLU por sus siglas en Ingles son: el $\mathrm{CO}_{2}, \mathrm{~N}_{2} \mathrm{O}$ y el $\mathrm{CH}_{4}$ (IPCC, 2006a) sin embargo por recomendaciones de la FAO en cultivos como la Quinoa no se considera el $\mathrm{CH}_{4}$. Asimismo, se define a la $\mathrm{HH}$ como "el volumen total de agua dulce usada para producir los bienes y servicios consumidos por el individuo o la comunidad o producidos por la empresa"(Hoekstra, Chapagain, Aldaya, \& Mekonnen, 2011) En cuanto la Huella Hídrica contiene tres componentes: Huella Verde (atribuida al agua lluvia), Huella Azul (Agua superficial y subterránea) y Huella Gris (contaminación de agua dulce) (Hoekstra et al., 2011).

El estudio tomo en cuenta el Ciclo de Vida de los cultivos de Quinoa, desde la preparación del suelo hasta la gestión de los residuos agrícolas, la investigación está basada en el inventario del sistema de producción agrícola; los datos se recolectaron mediante observaciones en campo, entrevistas con los productores, toma de muestra de suelo, fertilizantes, planta y raíces para su análisis posterior. Esta investigación se realiza con el objetivo de estimar la HC y $\mathrm{HH}$ del cultivo comunitario de Quinoa en los cantones de Cayambe-Pichincha-Ecuador (comunidad Pucará) y Colta-Chimborazo-Ecuador (comunidad Mjipamba), con el fin de conocer las emisiones de GEI y consumo de agua provenientes de la producción agrícola, datos necesarios para impulsar al eco-etiquetado de productos andinos ligado a la certificación orgánica que ya se cuenta en el país, contribuir con la mitigación del cambio climático y a un desarrollo sostenible.

\section{Materiales y Métodos}

El estudio se llevó acabo en los Cantones de Cayambe en la comunidad de Pucará y en Colta en la comunidad Majipamba, se identificaron 6 parcelas (Pucará) P1, P2, P3, P4 con un total 0,59 ha y 2 en Mijipamba C1 Y C2 con 0,26 ha, las parcelas fueron Georreferenciadas con el Dron Phantom 3 professional y con la ayuda del GPS Garmyn se tomaron los diferentes puntos de muestreo.

Se realizó entrevistas a los productores para obtener los datos de insumos utilizados para la producción, se establecieron una serie de preguntas Para recolectar información sobre: i) rendimientos, ii) cantidad y variedad de semilla utilizada en la siembra, iii) cantidad y tipos de combustibles usados en las maquinarias como: trilladora, venteadora, en el transporte todo terreno como el tractor y transporte terrestre (camioneta utilizada para el transporte de las panojas y maquinaria), iv) uso de fitosanitarios, v) aportaciones y procedencia de agua (si es de lluvia o sistemas de riego) y vi) las diferentes prácticas de manejo de cultivo.

Para reportar los datos de Huella de Carbono y Huella Hídrica la unidad funcional que se determinó fue un kilogramo de Quinoa producida. Para la toma de muestras se siguió la metodología de la guía dada por (FAO, 2008) y por el Instituto Nacional Autónomo de Investigaciones Agropecuarias (INIAP). Las muestras de suelo se tomaron mediante el método aleatorio simple siguiendo una trayectoria zig-zag.

\section{Estimación de emisiones de GEI}

El inventario de Gases de Efecto invernadero se realizó bajo las directrices dadas por el Grupo Intergubernamental 
de Expertos sobre el Cambio Climático (IPCC, 2006b) para el sector AFOLU y de Energía. Los datos fueron tabulados en Excel, el cual contiene los datos de actividad y factores de emisión, a su vez se tomó en cuenta los lineamientos protocolo de GEI desarrollado por World Resources Institute (WRI) y World Business Council for Sustainable Development (WBCSD) (2011). Para el Análisis de Ciclo de Vida se basó en la Norma Publicly Available Specification (PAS 2050:2011) desarrollada por British Standard Institute (BSI) (2011) y las recomendaciones de Ramirez-Cando et al., 2016.

\section{Estimación de GEI por el uso de combustibles}

$$
\text { Emisión }=\sum_{i=1}^{n} C i * E F i
$$

Emisión $=$ Emisiones de $\mathrm{CO}_{2}(\mathrm{~kg})$

Ci = Combustible vendido (TJ)

EFi $=$ Factor de emisión $(\mathrm{kg} / \mathrm{TJ})$. Es igual al contenido de carbono del combustible multiplicado por 44/12.

= Tipo de combustible (p. ej., gasolina, diésel, gas natural, GLP, etc.)

Fuente: (IPCC, 2006d).

Para las conversiones se utilizó los Valores Calóricos Netos (VCN) (TJ/Gg); Gasolina para motores 42,3 y diésel 43 (IPCC, 2006b), Factores de emisión por defecto para maquinaría todo terreno en la agricultura en $\mathrm{kg} / \mathrm{TJ} 74100$ $\mathrm{CO}_{2}, 4,15 \mathrm{CH}_{4}$ y $28,6 \mathrm{~N}_{2} \mathrm{O}$ (IPCC, 2006d) para el transporte terrestre que utilizan gasolina los factores usados fueron 69 $300 \mathrm{CO}_{2}, 8 \mathrm{CH}_{4}$ y $25 \mathrm{~N}_{2} \mathrm{O}$ y $74100 \mathrm{CO}_{2}, 3,9 \mathrm{CH}_{4}$ y $3,9 \mathrm{~N}_{2} \mathrm{O}$ para el diésel (IPCC, 2006d). la formula de estimacion se extiende a cada uno de los componentes del inventario y las emisiones producidas por la actividad.

\section{Estimación de GEI por emisiones directas de $\mathrm{N}_{2} \mathbf{0}$.}

Se tomaron las ecuaciones del capítulo 11 (IPCC, 2006c) para la estimación de emisiones directas de $\mathrm{N}_{2} \mathrm{O}$. De acuerdo el inventario en las zonas de estudio solo se aplica compost y no fertilizantes sintéticos por lo que las ecuaciones se resumen en:

$\mathrm{N}_{2}{ }_{\text {Directas }}^{\mathrm{O}}-\mathrm{N}=\mathrm{N}_{2} \mathrm{O}-\mathrm{N}_{\mathrm{N} \text { aportes }}$

\begin{aligned} \hline$N_{2}{ }^{O}$ Directas $^{-N} & =$ Emisiones directas anuales \\ & de $\mathrm{Ng} \mathrm{N}_{2} \mathrm{O}-\mathrm{N}$ producidas a partir \\ & de suelos gestionados \\ & $=$ Emisiones directas anuales $\mathrm{kg} \mathrm{N}_{2} \mathrm{O}-\mathrm{N}$ año \\ $N_{2} O-N_{\text {Naportes }} &$ de $\mathrm{N}_{2} \mathrm{O}-\mathrm{N}$ producidas \\ & por aportes de $\mathrm{N}$ a suelos \\ & gestionados \\ & $=$ cantidad anual de estiércol $\mathrm{kg} \mathrm{N}$ año-1 \\ & animal, compost, lodos \\ & cloacales y otros aportes de $\mathrm{N} \\ &$ aplicada a los suelos \end{aligned}

\begin{tabular}{ll} 
& $=$ cantidad anual del total de \\
& N de compost aplicada a los \\
$\boldsymbol{F}_{\text {сом }}$ & $\begin{array}{l}\text { suelos (asegurarse de que no } \\
\text { haya cómputo doble del N de } \\
\text { estiércol del compost) }\end{array}$ \\
\hline
\end{tabular}

Factor de emisión $=0,01\left[\mathrm{~kg} \mathrm{~N}_{2} \mathrm{O}-\mathrm{N}(\mathrm{kg} \mathrm{N})^{-1}\right](\mathrm{IPCC}$, 2006c)

$$
\operatorname{kg} \mathrm{N}_{2} \mathrm{O}=\mathrm{N}_{2} \mathrm{O}-\mathrm{N}_{\text {directas }} *(4 / 28)
$$

\section{Estimación de GEI por emisiones indirectas de} $\mathbf{N}_{2} \mathbf{0}$.

El $\mathrm{N}_{2} 0$ producido por la deposición atmosférica de $\mathrm{N}$ volatilizado de suelos gestionados $\left(\mathrm{N}_{2} \mathrm{O}_{\text {(ATD)- }} \mathrm{N}\right)$ se calculó mediante la ecuación 11.9 dada por e IPCC 2006.

$N_{2} O(A D T)-N=\left(F_{O N} * F_{r a c} c_{G A S M}\right) * E F_{4}$

Frac $_{\text {GASM }}$ es la "Fracción de materiales fertilizantes de $\mathrm{N}$ orgánico $\left(\mathrm{F}_{\mathrm{ON}}\right)$ y de $\mathrm{N}$ de orina y estiércol depositada por animales de pastoreo (FPRP) que se volatiliza como $\mathrm{NH}_{3}$ y NO. $\mathrm{kg} \mathrm{N}$ volatilizado ( $\mathrm{kg}$ de $\mathrm{N}$ aplicado o depositado)" (IPCC, 2006c). $\mathrm{EF}_{4}$ es el factor de emisión el cual equivale a $0,01\left[\mathrm{~kg} \mathrm{~N}-\mathrm{N}_{2} \mathrm{O}\right.$ $(\mathrm{kg} \mathrm{NH}-\mathrm{N}+\mathrm{NOx}-\mathrm{N}$ volatilizado) (IPCC, 2006c).

$$
\mathrm{kg} \text { de } \mathrm{CO}_{2} \text { eq }=\mathrm{kg} \text { de } \mathrm{N}_{2} \mathrm{O}^{*} 298
$$

\section{Estimación de Huella Hídrica}

La estimación de la Huella Hídrica para los cultivos de Quinoa se realizó con la ayuda del programa CROPWAT 8.0 (desarrollado por la FAO) y tabulaciones de datos en Excel, se sigue el procedimiento de cálculo mediante los manuales de la Water Footprint Network (WFN) y la Organización de la Naciones Unidas para la Agricultura y Alimentación (FAO).

Para el cálculo es necesario datos de:

\section{Tipo de cultivo:}

- Coeficiente de cultivo (Kc)

- Duración de cada fase (días)

- Profundidad radicular (m)

- Agotamiento crítico (p)

- Rendimiento productivo (Ky)

- Altura de cultivo (m)

\section{Datos de tipo de suelo:}

- Textura del suelo

- Humedad del suelo disponible total ( $\mathrm{mm} / \mathrm{m})$

- Tasa máxima de infiltración de precipitación $(\mathrm{mm} /$ día) 
- Profundidad radicular máxima (Zr, cm)

- Agotamiento inicial de humedad de suelo (\%)

\section{Evapotranspiración de referencia y precipitación efectiva.}

Se tomaron los datos meteorológicos: temperatura media mensual $\left({ }^{\circ} \mathrm{C}\right)$, humedad relativa media mensual $(\%)$, velocidad del viento-dirección predominante $(\mathrm{m} / \mathrm{s})$, Heliofanía media mensual (horas) y precipitación media mensual (mm) con información de 10 años (2000-2015) de las estaciones meteorológicas Tomalón-Tabacundo (Cayambe) y la estación Riobamba Politécnica (Riobamba) de Instituto de Meteorología e Hidrología (INAMHI). Para el caso de la Evapotranspiración de referencia (ETo) (mm/ día) se aplicó la fórmula de Penman-Monteith donde se tabularon con los datos de temperatura promedio $\left({ }^{\circ} \mathrm{C}\right)$, humedad $(\%)$, viento $(\mathrm{m} / \mathrm{s})$ e insolación (horas). La precipitación efectiva se realizó con la formula $\mathrm{FAO} /$ AGLW.

La ETc se tomó los datos de duración del periodo del cultivo de Quinoa y se consideró las condiciones de estrés hídrico.

$$
E T_{c}=K_{c} * E T_{o} \frac{m m}{\text { dia }}
$$

Donde:

$\mathrm{Kc}=$ coeficiente de cultivo (adimensional), indica la diferencia entre la evaporación y transpiración, donde integra las características del cultivo que presentan variación en cada fase. Para determinar este valor se siguió el Manual de la FAO de Evapotranspiración del cultivo. Los datos para estimar el Kc se tomaron los correspondientes a cereales de grano pequeño.

Se necesitó los datos de tipo de suelo, profundidad de raíces, valores de Agua Disponible Total (ADT), Agua Fácilmente Aprovechable (AFA) y nivel de agua en la zona de las raíces (Dr), siendo estos tres, los que permitieron calcular el dato del coeficiente; y cuyos valores, se describen dentro de la metodología de cálculo de Ks.

$$
E T_{c a j}=K_{s} * K_{c} * E T_{o}\left(\frac{m m}{\text { dia }}\right)
$$

Ks $=$ coeficiente de estrés hídrico (adimensional)

\section{Requerimientos de agua del cultivo \\ El Requerimiento de Agua del Cultivo verde (RAC verde)}

Dato de la ETc verde, para la fase 1 (fase inicial) correspondiente al área foliar que aún es pequeña y la ET que proviene de la evaporación del suelo, por lo que, es igual a la ETc (Allen, Pereira, Raes, \& Smith, 2006), las fases 2, 3 y 4 (fase de desarrollo, media y final) coinciden con la Precipitación efectiva ( $\mathrm{P}$ efec), cuando el valor no sobrepasa las necesidades hídricas del cultivo (Rodríguez, Garrido, Llamas, \& Varela, 2004).

\section{Requerimiento de Agua del Cultivo azul (RAC azul)}

Se representó como ETc azul, se estableció como una diferencia entre la ETc y la Precipitación efectiva; la cual representa el requerimiento de riego efectuado.

Se aplicó el factor de conversión donde:

$1 \mathrm{~mm} /$ día es equivalente a $10 \mathrm{~m}^{3} /$ ha*día (Allen et al., 2006)

Se obtuvo el Requerimiento de agua del cultivo (RAC) como se muestra en la ecuación:

$$
R A C=10 * E T_{C}\left(\frac{m^{2}}{h a}\right)
$$

\section{Requerimientos de agua del contaminante.}

el Requerimiento de Agua para eliminar los contaminantes (RAC gris), no se estimó ya que en las parcelas de estudio no existe la aplicación de productos químicos como: fertilizantes, plaguicidas, herbicidas, insecticidas que influencian, a generar residuos químicos en los cuerpos de agua dulce.

\section{Rendimientos productivos.}

Se aplicó la relación entre la cantidad cosechada de Quinoa (CC), expresado en toneladas (t) respecto al área de la parcela total $(\mathrm{S}, \mathrm{ha})$ el rendimiento se expresó en $(\mathrm{t} / \mathrm{ha})$.

$$
Y=\frac{c c}{s} \quad\left(\frac{t}{h a}\right)
$$

\section{Valor Huella Hídrica agrícola.}

El procedimiento de cálculo para obtener el valor de $\mathrm{HH}$, se diferenció el tipo de aportación de agua en las parcelas se estimó RAC azul (riego) $\left(\mathrm{m}^{3} / \mathrm{t}\right)$, y RAC verde para lluvia (precipitación).

\section{$\mathrm{HH}$ azul $=(\mathrm{RAC}$ azul $/ \mathrm{Y}) \quad \mathrm{HH}$ verde $=(\mathrm{RAC}$ verde $/ \mathrm{Y})$}

El valor de $\mathrm{HH}$ agrícola $\left(\mathrm{m}^{3} / \mathrm{t}\right)$ se obtuvo mediante la siguiente ecuación:

\section{Resultados y discusión}

\section{Resultados}

En la Tabla 1 se muestran los datos del inventario de entradas y salidas recolectados para la estimación de la huella de Carbono de acuerdo a las entrevistas realizadas a los productores y las observaciones realizadas en campo, dentro de los datos necesarios para la estimación se tiene que la productividad en Mijipamba es 2,03 t/ha y 1,66 t/ ha en Pucara. Debido a la alta producción de Pucara se genera 2,8 t/ha de residuo después de realizar la trilla y en Mijipamba se produce 2,5 t/ha otros de los inputs que se utilizaron para la estimación es el uso de combustibles, semillas, cantidad de fertilizante y composición del mismo, se consideraron los datos de fitosanitarios, pero para las dos zonas de estudio no se aplica ninguna dosis. 
Tabla 1. Datos de Entradas y Salidas en la fase cultivo, referido a la producción de un kilogramo de Quinoa fresca

\begin{tabular}{lcccc}
\hline \multicolumn{4}{c}{ Descripción } & \multicolumn{4}{c}{ Cantidad } & Unidades \\
\hline \multirow{5}{c}{ Mijipamba } & Pucara \\
Mano de & Personas & 0,320 & 0,242 & Personas*Horas/ha \\
\cline { 2 - 5 } & & 3,940 & 4,919 & $\mathrm{~kg} / \mathrm{ha}$ \\
Fertilizantes & Nitrógeno & 0,006 & 0,007 & $\mathrm{~kg} / \mathrm{ha}$ \\
Orgánicos & Fósforo & 0,002 & 0,002 & $\mathrm{~kg} / \mathrm{ha}$ \\
(compost) & Potasio & 0,002 & 0,002 & $\mathrm{~kg} / \mathrm{ha}$ \\
& Diésel & 0,041 & 0,024 & $\mathrm{~kg} / \mathrm{ha}$ \\
Combustible & Gasolina & 0,011 & 0,018 & $\mathrm{~kg} / \mathrm{ha}$ \\
Semillas & - & 0,009 & 0,009 & $\mathrm{~kg} / \mathrm{ha}$ \\
Fitosanitarios & Pesticidas & 0,000 & 0,000 & $\mathrm{~kg} / \mathrm{ha}$ \\
\hline \multicolumn{5}{c}{ Outputs } \\
\hline $\begin{array}{c}\text { Granos de } \\
\text { Quinoa }\end{array}$ & 1,000 & 1,000 & $\mathrm{~kg} / \mathrm{ha}$ \\
$\begin{array}{c}\text { Residuos de } \\
\text { trilla }\end{array}$ & 1,531 & 1,377 & $\mathrm{~kg} / \mathrm{ha}$ \\
\hline
\end{tabular}

\section{Resultados de parámetros Físicos-Químicos Clase textural}

De acuerdo a los porcentajes de arena, arcilla y limo como se muestran en la Tabla 2 y según la clasificación USDA los suelos muestreados presentan una clase textural franco arcilloso arenoso, considerándolos suelos francos con textura moderadamente fina.

Tabla 2. Clases texturales

\begin{tabular}{lllll}
\hline Parcelas & \% Arena & \% Arcilla & \% Limo & Clase Textural \\
\hline P1 & 66 & 28 & 6 & Franco Arcilloso Arenoso \\
P2 & 64 & 29 & 7 & Franco Arcilloso Arenoso \\
P3 & 63 & 31 & 6 & Franco Arcilloso Arenoso \\
P4 & 61 & 33 & 6 & Franco Arcilloso Arenoso \\
C1 & 56 & 33 & 11 & Franco Arcilloso Arenoso \\
C2 & 60 & 31 & 9 & Franco Arcilloso Arenoso \\
\hline
\end{tabular}

Tabla 3. Contenido de Carbono y Materia orgánica en las parcelas en Pucara

\begin{tabular}{cccc}
\hline Parcela & $\begin{array}{c}\text { Carbón } \\
\text { Orgánico } \\
(\mathrm{mg} / \mathrm{g})\end{array}$ & $\begin{array}{c}\text { \% Carbón } \\
\text { Orgánico } \\
\text { COS }\end{array}$ & $\begin{array}{c}\text { \% Materia } \\
\text { orgánica MO }\end{array}$ \\
\hline P1 & 28,00 & 2,80 & 3,64 \\
P2 & 22,74 & 2,27 & 2,96 \\
P3 & 16,85 & 1,68 & 2,19 \\
P4 & 25,64 & 2,56 & 3,33 \\
P-RAÍZ & 87,10 & 8,71 & 11,32 \\
C1 & 18,59 & 1,86 & 2,42 \\
C2 & 22,59 & 1,76 & 2,94 \\
C-RAÍZ & 136,65 & 14,49 & 17,77 \\
\hline
\end{tabular}

\section{Carbón Orgánico y Materia Orgánica.}

La Tabla 3 se muestra los porcentajes de Carbón Orgánico y Materia Orgánica encontrada en los suelos de Cayambe en un rango de 1,68 -2,80 \% Carbón Orgánico y 2,19 a $3,64 \%$ de MO y en Mijipamba con 1,86-2,26 \% de Carbón Orgánico y 2,42 a 2,94 \% de MO. En las raíces se encontró 11,32 de MO y 17,77 de MO en Cayambe y Mijipamba respectivamente.

\section{Nitrógeno Total}

El contenido de Nitrógeno total por hectárea aplicado a los suelos de Mijipamba y Pucará se muestra en la Tabla 4. En Pucará se estimó que la cantidad aplicada es de $15 \mathrm{~kg} \mathrm{~N} / \mathrm{ha}$ y en Mijipamba 9,9 kg N/ha.

Tabla 4. Contenido de Nitrógeno Total $\mathrm{kg} \mathrm{N} / \mathrm{ha}$

\begin{tabular}{lllll}
\hline \multicolumn{1}{c}{ Tipo de muestra } & \multicolumn{1}{c}{ Pucará } & \multicolumn{2}{c}{ Mijipamba } \\
\hline Fertilizante (Compost) & 10 & $\mathrm{t} / \mathrm{ha}$ & 6,6 & $\mathrm{t} / \mathrm{ha}$ \\
N total & 1500 & $\mathrm{mg} / \mathrm{kg}$ & 1500 & $\mathrm{mg} / \mathrm{kg}$ \\
& 15 & $\mathrm{~kg} \mathrm{~N} / \mathrm{ha}$ & 9,9 & $\mathrm{~kg} \mathrm{~N} / \mathrm{ha}$ \\
\hline
\end{tabular}

\section{Resultado de la Huella de Carbono en los cultivos de Quinoa}

En la Tabla 5 se muestra los resultados obtenidos de la Huella de Carbono en Pucara y en Mijipamba, se reporta $468,54 \mathrm{~kg} \mathrm{CO}_{2}$ eq $/$ ha y $425,38 \mathrm{~kg} \mathrm{CO}_{2}$ eq/ha respectivamente, donde el uso de los combustibles contribuye con $71 \%$ de las emisiones de GEI en Mijipamba y 60,70 \% en Pucara, esto se debe principalmente por el diésel usado por el tractor con 172,86 kg CO eq/ha en Pucara y 216,073 $\mathrm{kg} \mathrm{CO}$ eq/ha en Mijipamba en la fase preparación del suelo. (Figura 2). El uso y producción de Fertilizantes Orgánicos aplicados al suelo aporta con el 28\% en Mijipamba y $38 \%$ en Pucara (Figura 3). Esto se ve reflejado por la aplicación de $\mathrm{N}$ en forma de fertilizante orgánico, se estima que las emisiones directas de $\mathrm{N}_{2} \mathrm{O}$ para Pucara es de 70,24 $\mathrm{kgCO}_{2}$ eq/ha y para Mijipamba las emisiones son más bajas con $46,36 \mathrm{kgCO}_{2}$ eq/ha, ya que en Pucara aplican 10 t de compost y en Mijipamba 6 t. Las emisiones indirectas de $\mathrm{N}_{2} \mathrm{O}$ por la deposición atmosférica de $\mathrm{N}$ volatilizado de los suelos gestionados en Pucara y Mijipamba son 14,05 y 9,27 $\mathrm{kgCO}_{2}$ eq/ha respectivamente. (Figura 4).

De acuerdo al análisis del ciclo de vida ACV de la Quinoa la fase preparación del suelo es donde se emite la mayor parte de GEI con un $79 \%$ en Mijipamba y $75 \%$ en Pucara, esto se debe al combustible por parte del tractor para el arado del suelo y la cantidad de compost utilizado para la fertilización del suelo. la fase de la cosecha se reporta emisiones por el uso de la trilladora la cual funciona a base de diésel o gasolina y los vehículos que utilizan para transportar las panojas de Quinoa aportándose en esta fase en Mijipamba y Pucara 29,91\% y 24\% respectivamente. 
Temisiones de gei por la producción de quinoa por fuente de emisión.

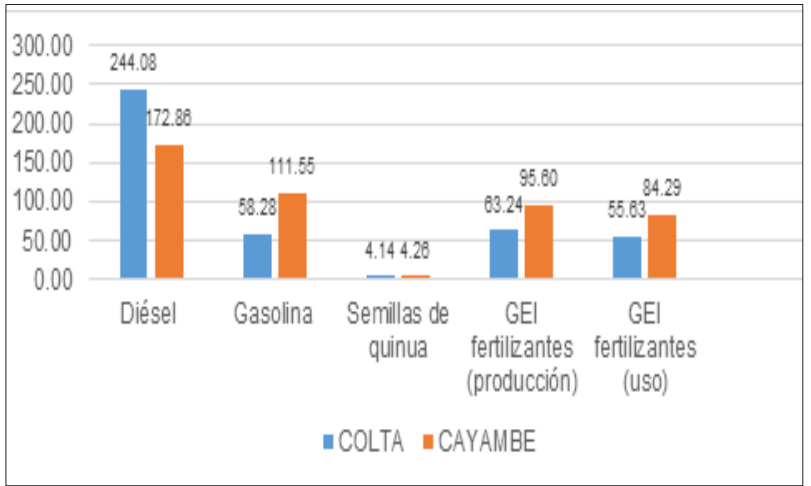

Figura 3. Emisiones GEI por fuente de emisión en la producción de Quinoa en Pucara y Mijipamba

Emisiones directas e indirectas de $\mathrm{N}_{2} \mathrm{O}$

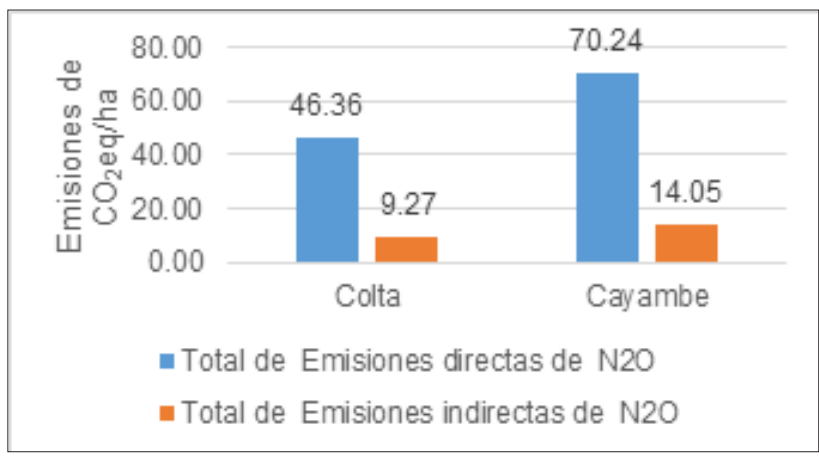

Figura 4. Emisiones Directas e Indirectas de $\mathrm{N}_{2} 0$ por la acción biológica

\section{Huella hídrica}

En Pucara para la etapa media y final, las plantas tenían una altura promedio de 0,90 y $1,50 \mathrm{~m}$, respectivamente; mientras que, para las etapas media y final, presentaban un tamaño de raíz de 0,50 y 1,50 m. En Mijipamba las plantas presentaron una altura promedio de $2,65 \mathrm{~m}$ para la etapa media y $2,00 \mathrm{~m}$ para la etapa final; la raíz entre la etapa media y final fue de 0,80 y $1,50 \mathrm{~m}$ (Tabla 6).

\section{Evapotranspiración y RAC por componentes de HH}

Las tres componentes verde, azul y gris de $\mathrm{HH}\left(\mathrm{m}^{3} / \mathrm{t}\right)$ se presentan en la Tabla 7, los valores de ET gris se consideraron como $0,00 \mathrm{~mm} /$ período, al no existir aplicaciones de productos químicos.

\section{Huellas Hídricas Verde, Azul y Gris}

En Pucara la dotación de agua se realizaba por sistemas de riego por aspersión, y en Mijipamba por lluvia (precipitación). Se identificó que los mayores resultados en el caso de Pucará para $\mathrm{HH}$ azul y verde $\left(\mathrm{m}^{3} / \mathrm{t}\right)$ fueron en $\mathrm{P} 3$ con $595,49 \mathrm{~m}^{3} / \mathrm{t}$ y $311,25 \mathrm{~m}^{3} / \mathrm{t}$, respectivamente; donde, los valores de $\mathrm{HH}$ azul superaron los de $\mathrm{HH}$ verde.
Y para las parcelas de Mijipamba, aunque, se dotaban de agua por lluvia, los mayores valores que tuvieron fueron $\mathrm{C} 1$ con una $\mathrm{HH}$ azul y verde $\left(\mathrm{m}^{3} /\right.$ ton $)$ de $82,10 \mathrm{~m}^{3} / \mathrm{t}$ y 39,54 $\mathrm{m}^{3} / \mathrm{t}$. Sin embargo, la dimensión de los resultados de $\mathrm{HH}$ azul que se obtuvieron en Pucará, fueron superiores a los de Mijipamba, por una amplia diferencia; dado que, la primera realiza dotación por sistema de riego y por ello, consumió mayor cantidad de agua.

Finalmente, los resultados de $\mathrm{HH}$ gris $\left(\mathrm{m}^{3} / \mathrm{t}\right)$ por parcela, se reportaron como nulos o cero $\left(0 \mathrm{~m}^{3} / \mathrm{t}\right)$; debido a que, en ambas comunidades en los cultivos no se aplican dosis de fitosanitarios. (Tabla 8)

\section{Huella Hídrica agrícola}

Los resultados que se presentan en la Tabla 9, pertenecen a la $\mathrm{HH}$ agrícola $\left(\mathrm{m}^{3} /\right.$ ton $)$ por parcela, siendo las necesidades de agua de los cultivos para obtener donde se indica que: $\mathrm{P} 1$ presentó $330,87 \mathrm{~m}^{3} / \mathrm{t}$; P2 tuvo $138,10 \mathrm{~m}^{3} / \mathrm{t}$ de P3 se obtuvo $906,84 \mathrm{~m}^{3} / \mathrm{t}$ y $\mathrm{P} 4$ unos $50,25 \mathrm{~m}^{3} / \mathrm{t}$ para Pucará; en cambio, en Mijipamba fueron en $\mathrm{C} 1 \mathrm{de} 121,64 \mathrm{~m}^{3} / \mathrm{t}$ y $\mathrm{C} 2$ de $75,33 \mathrm{~m}^{3} / \mathrm{t}$.

Emisiones por cada fase de cultivo de la quinoa

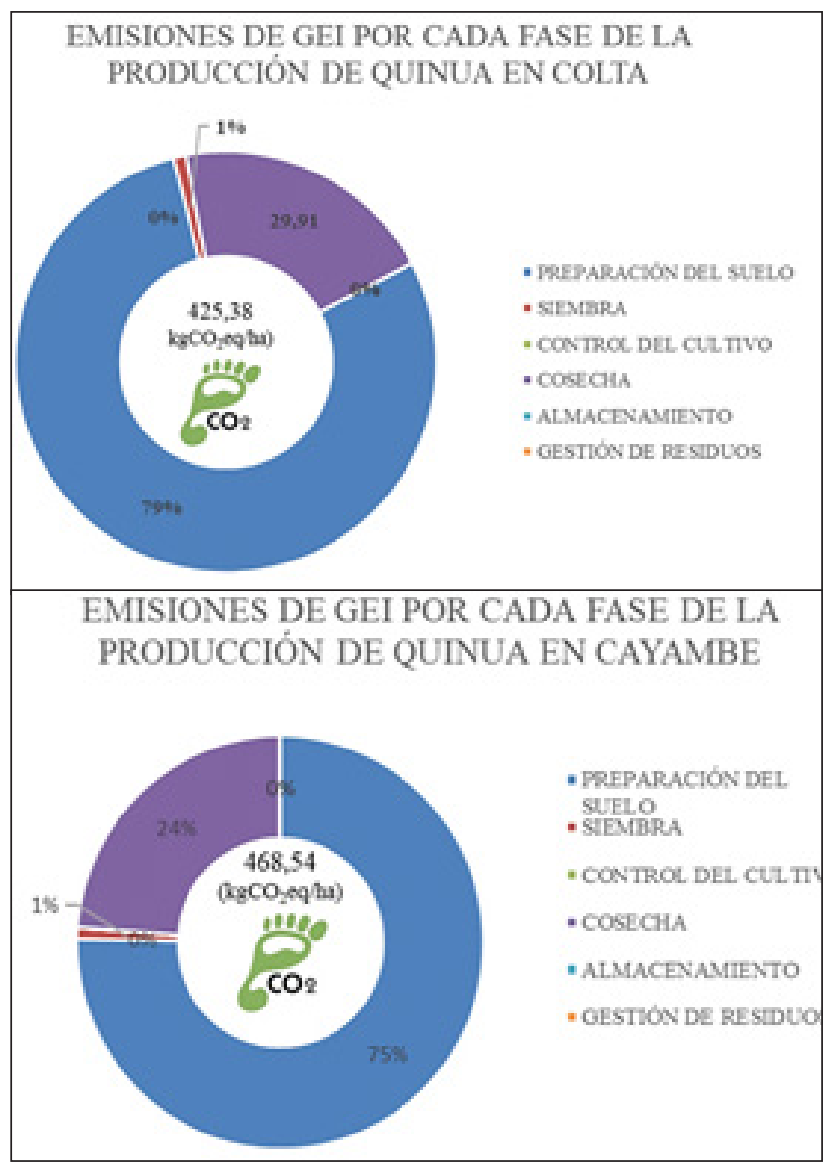

Figura 5. Emisiones por cada fase producción de Quinoa, se muestra que la fase de preparación del suelo es donde se emite mayoritariamente con las emisiones 
Tabla 5. Huella de Carbono total y referida a la unidad funcional

\begin{tabular}{|c|c|c|c|c|c|c|c|c|}
\hline \multirow[b]{2}{*}{ Entradas INPUT } & \multicolumn{4}{|c|}{ MIJIPAMBA } & \multicolumn{4}{|c|}{ PUCARA } \\
\hline & 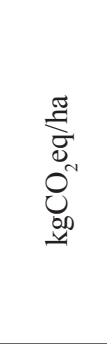 & 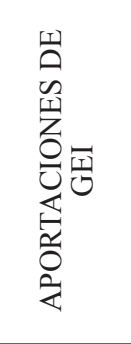 & 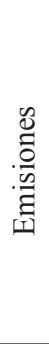 & 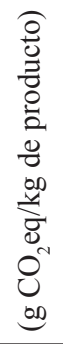 & 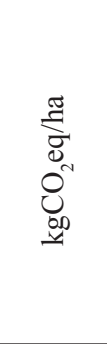 & 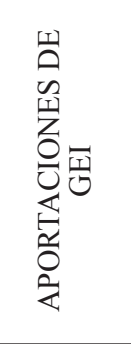 & 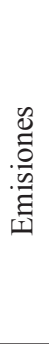 & $\begin{array}{l}0 \\
0 \\
0 \\
0 \\
0 \\
0 \\
0 \\
0 \\
0 \\
0 \\
0 \\
0 \\
0 \\
0 \\
0 \\
0\end{array}$ \\
\hline Combustibles & 302,36 & $71,08 \%$ & & & 284,40 & $60,70 \%$ & & 83 \\
\hline Semillas de Quinoa & 4,14 & $0,97 \%$ & & & 4,24 & $0,91 \%$ & & 99 \\
\hline GEI fertilizantes (producción) & 63,24 & $14,87 \%$ & & & 95,60 & $20,40 \%$ & & 01 \\
\hline GEI fertilizantes (uso) & 55,63 & $13,08 \%$ & & & 84,29 & $17,99 \%$ & & \\
\hline Total & 425,38 & $100,00 \%$ & & 16 & 468,56 & $100,00 \%$ & & 38 \\
\hline
\end{tabular}

Tabla 6. Características físicas de las estructuras vegetales de Quinoa en las parcelas estudiadas

\begin{tabular}{|c|c|c|c|c|c|c|c|c|c|}
\hline \multirow[b]{2}{*}{ Características } & \multirow[b]{2}{*}{ Abertura } & \multicolumn{5}{|c|}{ PUCARA } & \multicolumn{3}{|c|}{ MIJIPAMBA } \\
\hline & & P1 & $\mathrm{P} 2$ & $\mathrm{P} 3$ & P4 & Promedio & $\mathrm{C} 1$ & $\mathrm{C} 2$ & Promedio \\
\hline \multirow{2}{*}{ Altura de planta $(\mathrm{h}, \mathrm{m})$} & Etapa media & 0,86 & 0,92 & 0,90 & 0,91 & 0,90 & 1,80 & 1,50 & 1,65 \\
\hline & Etapa final & 1,50 & 1,50 & 1,50 & 1,50 & 1,50 & 2,00 & 2,00 & 2,00 \\
\hline \multirow{2}{*}{ Tamaño de raíz (m) } & Etapa media & 0,50 & 0,50 & 0,50 & 0,50 & 0,50 & 0,80 & 0,80 & 0,80 \\
\hline & Etapa final & 1,50 & 1,50 & 1,50 & 1,50 & 1,50 & 1,50 & 1,50 & 1,50 \\
\hline
\end{tabular}

Tabla 7. Evapotranspiración y RAC de las componentes de HH por parcela

\begin{tabular}{ccccccc}
\hline Parcelas & $\begin{array}{c}\text { ET verde }(\mathrm{mm} / \\
\text { período })\end{array}$ & $\begin{array}{c}\text { ET azul } \\
(\mathrm{mm} / \text { período })\end{array}$ & $\begin{array}{c}\text { ET gris } \\
(\mathrm{mm} / \text { período })\end{array}$ & $\begin{array}{c}\text { RAC verde } \\
\left(\mathrm{m}^{3} / \mathrm{ha}\right)\end{array}$ & $\begin{array}{c}\text { RAC azul } \\
\left(\mathrm{m}^{3} / \mathrm{ha}\right)\end{array}$ & $\begin{array}{c}\text { RAC gris } \\
\left(\mathrm{m}^{3} / \mathrm{ha}\right)\end{array}$ \\
\hline PUCARÁ & & & Período $2000-2015$ & & \\
P1 & 11,39 & 41,54 & 0,00 & 113,95 & 415,45 & 0,00 \\
P2 & 12,68 & 29,35 & 0,00 & 126,79 & 293,51 & 0,00 \\
P3 & 15,13 & 28,94 & 0,00 & 151,26 & 289,38 & 0,00 \\
P4 & 9,79 & 40,47 & 0,00 & 97,86 & 404,65 & 0,00 \\
\hline MIJIPAMBA & & \multicolumn{7}{c}{ Período 2006-2015 } \\
C1 & 10,41 & 21,61 & 0,00 & 104,06 & 216,06 & 0,00 \\
C2 & 10,41 & 21,87 & 0,00 & 104,09 & 218,74 & 0,00 \\
\hline
\end{tabular}

Tabla 8. Resultados de HH verde, azul y gris en $\left(\mathrm{m}^{3} / \mathrm{t}\right)$ por parcela

\begin{tabular}{lllllll}
\hline Parcelas & $\begin{array}{c}\text { RAC verde } \\
\left(\mathrm{m}^{3} / \mathrm{ha}\right)\end{array}$ & $\begin{array}{c}\text { RAC azul } \\
\left(\mathrm{m}^{3} / \mathrm{ha}\right)\end{array}$ & $\begin{array}{c}\text { RAC gris } \\
\left(\mathrm{m}^{3} / \mathrm{ha}\right)\end{array}$ & $\begin{array}{c}\text { HH verde } \\
\left(\mathrm{m}^{3} / \mathrm{t}\right)\end{array}$ & $\begin{array}{c}\text { HH azul } \\
\left(\mathrm{m}^{3} / \mathrm{t}\right)\end{array}$ & $\begin{array}{c}\text { HH gris } \\
\left(\mathrm{m}^{3} / \mathrm{t}\right)\end{array}$ \\
\hline P1 & 113,95 & 415,45 & 0,00 & 71,22 & 259,65 & 0,00 \\
P2 & 126,79 & 293,51 & 0,00 & 41,66 & 96,44 & 0,00 \\
P3 & 151,26 & 289,38 & 0,00 & 311,25 & 595,49 & 0,00 \\
P4 & 9,79 & 40,47 & 0,00 & 9,79 & 40,47 & 0,00 \\
\hline C1 & 104,06 & 216,06 & 0,00 & 39,54 & 82,10 & 0,00 \\
C2 & 104,09 & 218,74 & 0,00 & 24,29 & 51,04 & 0,00 \\
\hline
\end{tabular}


Tabla 9. Resultados de Huella Hídrica (m3/ton) agrícola por parcela

\begin{tabular}{ccccc}
\hline Parcelas & $\begin{array}{c}\text { HH verde } \\
\left(\mathrm{m}^{3} / \mathrm{t}\right)\end{array}$ & $\begin{array}{c}\mathrm{HH} \text { azul } \\
\left(\mathrm{m}^{3} / \mathrm{t}\right)\end{array}$ & $\begin{array}{c}\mathrm{HH} \text { gris } \\
\left(\mathrm{m}^{3} / \mathrm{t}\right)\end{array}$ & $\begin{array}{c}\mathrm{HH} \text { agrícola } \\
\left(\mathrm{m}^{3} / \mathrm{t}\right)\end{array}$ \\
\hline P1 & 71,22 & 259,65 & 0,00 & 330,87 \\
P2 & 41,66 & 96,44 & 0,00 & 138,10 \\
P3 & 311,25 & 595,49 & 0,00 & 906,84 \\
P4 & 9,79 & 40,47 & 0,00 & 50,26 \\
\hline Total & 433,92 & 992,05 & 0,00 & 1426,07 \\
\hline C1 & 39,54 & 82,10 & 0,00 & 121,64 \\
C2 & 24,29 & 51,04 & 0,00 & 75,33 \\
\hline Total & 63,83 & 133,14 & 0,00 & 196,97 \\
\hline \multirow{2}{*}{ Total } & HH verde & $\begin{array}{c}\mathrm{HH} \text { azul } \\
\left(\mathrm{m}^{3} / \mathrm{t}\right)\end{array}$ & $\begin{array}{c}\mathrm{HH} \text { gris } \\
\left(\mathrm{m}^{3} / \mathrm{t}\right)\end{array}$ & $\begin{array}{c}\mathrm{HH} \text { agrícola } \\
\left(\mathrm{m}^{3} / \mathrm{t}\right)\end{array}$ \\
\hline & 497,75 & 1125.19 & 0,00 & 1623,04 \\
\hline
\end{tabular}

Los resultados presentados de la Figura 6, pertenecen a la $\mathrm{HH}$ agrícola $\left(\mathrm{m}^{3} / \mathrm{t}\right)$ promedio se obtuvo un valor de $\mathrm{HH}$ de $356,49 \mathrm{~m}^{3} / \mathrm{t}$ para Pucara y $98,49 \mathrm{~m}^{3} / \mathrm{t}$ para Mijipamba.

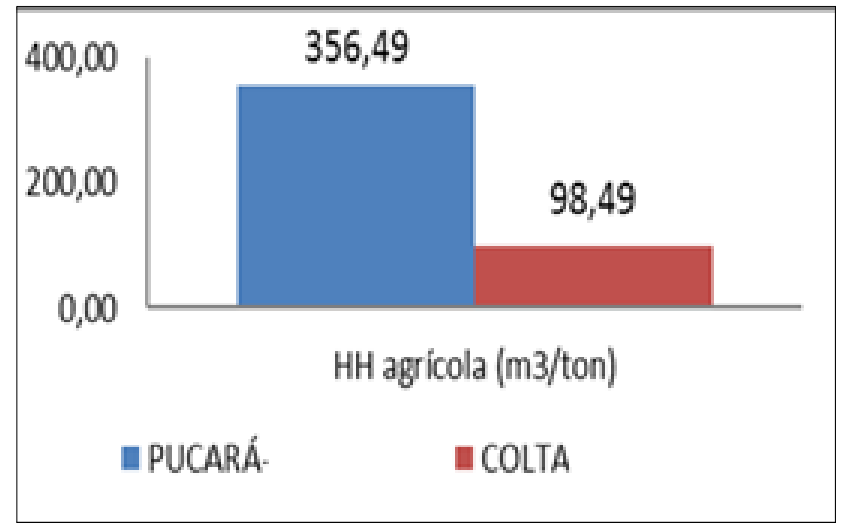

\begin{tabular}{lc}
\hline Comunidad & $\begin{array}{c}\text { HH agrícola }\left(\mathrm{m}^{3} / \mathrm{t}\right) \text { promedio } \\
\text { por comunidad }\end{array}$ \\
\hline Pucará & 356,49 \\
\hline Mijipamba & 98,49 \\
\hline Total & 454,98 \\
\hline
\end{tabular}

Figura 6. Resultados de Huella Hídrica promedio $\left(\mathrm{m}^{3} / \mathrm{t}\right)$ agrícola por comunidad

\section{Discusión}

Los factores edáficos como textura y materia orgánica determinaron que los suelos de las zonas en estudio representan un depósito de Carbono que contribuyen a la absorción de $\mathrm{CO}_{2}$ de la atmosfera. En Pucara los suelos contiene entre 2,19 a 3,64\% de MO, y en Riobamba entre 2,42 a 2,94 \% de MO, según el análisis de suelos dado por Rioja 2002 estos rangos corresponden a un nivel normal a muy alto contenido de $\mathrm{MO}$; determinando un secuestro de carbono efectivo, según el estudio Mogollon, Rivas, Martinez, Campos, \& Marquez (2015), el suelo es considerado un reservorio importante del carbono a escala global, si no el más importante (Ramiréz Cando \& Spugnoli, 2016). La investigación de Carvajal (2010) determinó que los suelos tienen la capacidad de almacenar Carbón por el material vegetal acumulado en descomposición y que existe pérdida de carbono cuando se retira del cultivo o se quema, en el caso de la Quinoa esto es coherente con el manejo que le dan las comunidades, incorporando la biomasa residual al suelo, sin quemarla.

La HC que se estimó en Pucara fue de 458,56 $\mathrm{kgCO}_{2}$ eq/ ha $\mathrm{y}$ en Mijipamba de 425,38 $\mathrm{kgCO}_{2}$ eq/ha, de acuerdo al estudio realizado por Altuna et al., (2012), donde se estiman la $\mathrm{HC}$ en cereales como el trigo, la cebada y el maíz, reportan $380,87,297,75$ y 514,76 gCO 2 eq $/ \mathrm{kg}$ de producto respectivamente, comparando con la $\mathrm{HC}$ de la Quinoa, se evidencia valores más bajos de emisiones entre $44 \%$ a $50 \%$ que los cereales mencionados, con una $\mathrm{HC}$ de 230,38 g CO eq por cada $\mathrm{kg}$ de Quinoa producido en Pucara y 256,16 $\mathrm{g} \mathrm{CO}_{2}$ eq $/ \mathrm{kg}$ en Mijipamba, es importante destacar que la reducción en las emisiones responde mayoristamente al rendimiento mayor en Pucara.

En el caso de estudio de Altuna et al. Las fuentes que contribuyen principalmente a las emisiones de GEI, es el $\mathrm{N}_{2} \mathrm{O}$ emitido a la atmosfera por el uso de fertilizantes inorgánicos en un $80 \%$ seguido por el uso de combustibles, a diferencia del caso de producción de Quinoa, donde la mayor contribución se debe al uso de combustibles, aportando entre $61-71 \%$ de las emisiones, seguido de las aportaciones por la fertilización orgánica que representan el $28-40 \%$. Algo para destacar junto con el uso de fertilizantes orgánicos, es que, la Quinoa en las zonas de estudios no requiere fitosanitarios.

En cuanto la $\mathrm{HH}$ los resultados de las componentes azul y verde, demostraron una inclinación hacia la $\mathrm{HH}$ azul para ambas comunidades, presentando un consumo bajo de aproximadamente un $30 \%$ de $\mathrm{HH}$ verde en las mismas; esto se debe, a que la aplicación de $\mathrm{HH}$ azul en los cultivos, favorece el aumento del rendimiento; pudiendo diferenciarse con los resultados de las entidades (Pegasys Strategy and Development (PTY) LTD, WWF, Universidad Nacional Agraria La Molina UNALM, \& Autoridad Nacional del Agua - ANA, 2015) obtenidos en Perú, donde la $\mathrm{HH}$ verde representa el $85 \%$ de sus resultados finales; sin embargo, en la mayoría de las parcelas de estudio, la aportación de agua se da por sistema de riego, como el caso de Pucará; mientras que en Mijipamba, los resultados de $\mathrm{HH}$ azul corresponden a los que tendrían las parcelas si estas tuvieran aportación de riego; es por esto, que la cantidad de $\mathrm{HH}$ verde generada en 0,26 ha es de solo $63,83 \mathrm{~m} 3 / \mathrm{t}$ a diferencia de $\operatorname{los} 3665 \mathrm{~m} 3 / \mathrm{t}$ producidos en toda la extensión territorial de cultivos de Quinoa en Perú; indicando un resultado aceptable; por lo que, es necesario ampliar este valor respecto a las hectáreas de cultivo a nivel nacional, para obtener un resultado más óptimo para la comparación.

Según (Geerts et al., 2009) en el Estado del Arte de la Quinoa en el Mundo presentado por la FAO en 2013; los resultados para Quinoa del altiplano boliviano, en base con 
la capacidad de soportar el estrés hídrico, que relacionan al rendimiento por consumo de agua, arrojan valores entre 0,3 y $0,6 \mathrm{~kg} / \mathrm{m}^{3}$; y que por conversión de unidades son iguales a 3333 y $6666 \mathrm{~m}^{3} / \mathrm{t}$; van a ser superiores al valor obtenido en conjunto entre las comunidades.

Por último, (Mekonnen \& Hoekstra, 2010) en los reportes de la WFN para cultivos, presentan el valor de $\mathrm{HH}$ verde de Quinoa en Ecuador es igual a $5545 \mathrm{~m}^{3} / \mathrm{t}$; por este motivo, la investigación se enfocó en generar datos en los cantones de Pucara y Mijipamba, considerando su gran nivel de producción de Quinoa y que presentan valores para las componentes verde y azul en el sector agrícola, que son iguales a 497,75 y $1125,19 \mathrm{~m}^{3} / \mathrm{t}$, respectivamente; donde el valor de $\mathrm{HH}$ verde obtenido en conjunto representa solo un $9 \%$ al reportado por la WFN. Los resultados obtenidos en este estudio, generan información que representa el consumo de agua en los cantones y a nivel nacional; además, de poder diferenciar el tipo de dotación que se aplica en las parcelas.

Por lo anterior dicho, se puede deducir que la mayor aportación para el resultado final, es dado por la generación de $\mathrm{HH}$ azul presente en las comunidades, sobre todo por las parcelas de Pucará, que, a su vez, representan casi el $70 \%$ de la superficie, rendimiento y resultados de todo el estudio; mientras que las de Mijipamba, representan el consumo de $\mathrm{HH}$ verde y el posible de $\mathrm{HH}$ azul si aplicaran riego.

\section{Conclusiones}

En base a los resultados obtenidos de la estimación de Huella de Carbono en los cultivos de Quinoa de los cantones de Pucara y Mijipamba, se reporta que las emisiones significativas se presentan en la de fases de preparación del suelo y la cosecha, atribuido al uso combustible por parte de la maquinaria, transporte todo terreno y terrestre. Otra de las fuentes que emite considerables cantidades de emisiones de GEI son los fertilizantes orgánicos con emisiones directas e indirectas de $\mathrm{N}_{2} \mathrm{O}$. Es importante considerar que los agricultores imparten un manejo agronómico orgánico, por lo que, los suelos no presentan erosión y contienen un alto contenido de materia orgánica, considerándolo un depósito de carbono efectivo para la absorción de $\mathrm{CO}_{2}$ de la atmosfera y con esto estarían neutralizando la Huella estimada.

En cuanto a la Huella Hídrica, se reportó que para la producción de Quinoa en Pucara depende del agua almacenamiento (componente azul) aplicada por riego y al agua de lluvia, la cual aporta una mayor cantidad a la obtención del rendimiento; sin embargo, en Mijipamba se obtuvo una producción únicamente a base de las condiciones climáticas, teniendo valores de $\mathrm{HH}$ verde bajos pero aceptables, ya que, los datos de $\mathrm{HH}$ azul que se obtienen, son datos estimados si en la comunidad existiera la aportación de agua por riego.

Utilizando las herramientas de ACV, Huella de Carbono y Huella Hídrica se podría lograr las certificaciones con ecoetiquetas de los productos andinos los cuales son apetecidos en países europeos ya que la Quinoa es un producto muy apetecido por sus altos valores nutritivos y es un producto que el Ecuador exporta bajo la certificación orgánica.

\section{Literatura citada}

Allen, R. G.; Pereira, L. S.; Raes, D. \& Smith, M. 2006. Evapotranspiración del cultivo. Guías para la determinación de los requerimientos de agua de los cultivos. Estudio de FAO de riego y drenaje (Vol. 56). Roma.

Altuna, A.; Intia, L.; Hierro, O.; Unamunzaga, O.,;Neiker, B. \& Ucan, F. D. 2012. Huella de carbono de los cereales análisis de la emisión de gases de efecto invernadero en el sector agroalimentario (pp. 31-38). Navarra Agraria.

BSI. 2011. Publicly Available Specification PAS 2050: 2011 Specification for the assessment of the life cycle greenhouse gas emissions of goods and services. System. http://doi.org/978 0580713828.

Carvajal, M. 2010. Absorción de $\mathrm{CO}_{2}$ por los cultivos más representativos. Consejo Superiro de Investigaciones Cientificas, 1, 43.

CEPAL. 2012. Huella de carbono y exportaciones de alimentos. Guía práctica. Santiago de Chile.

FAO. 2008. Guide to laboratory establishment for plant nutrient analysis. Roma.

FAO. 2013. Valor nutricional- Año Internacional de la Quinua 2013 un futuro sembrado hace miles de años.

FAO. (2017). Quinua.

FAO, \& ALADI. 2014. Tendencias y perspectivas del comercio internacional de quinua. Santiago. a Organización de las Naciones Unidas para la Alimentación y la Agricultura en colaboración con la Asociación Latinoamericana de Integración Las. http:// doi.org/10.1016/S1043-4526(09)58001-1.

Hoekstra, A., Chapagain, A., Aldaya, M., \& Mekonnen, M. 2011. The Water Footprint Assessment Manual: Setting the Global Standard. Water Footprint Network.

IPCC. 2006a. Capítulo 1: Introducción. In S. Eggleston, L. Buendia, K. Miwa, T. Ngara, \& K. Tanabe (Eds.), Directrices del IPCC 2006 para los Inventarios Nacionales de Gases de Efecto Invernadero Volumen 4 Agricultura, Silvicultura y Otros Usos de la Tierra. (pp. 1-25). Japón: IGES.

IPCC. 2006b. Capítulo 1: Introducción. In Directrices del IPCC de 2006 para los Inventarios Nacionales de Gases de Efecto Invernadero Volumen 2. Energía (pp. 1-30). Japón: IGES.

IPCC. 2006c. Capítulo 11: Emisiones de $\mathrm{N}_{2} \mathrm{O}$ de los suelos gestionados y emisiones de $\mathrm{CO}_{2}$ derivadas de la aplicaciónde cal y urea. In S. Eggleston, L. Buendia, K. Miwa, T. Ngara, \& K. Tanabe (Eds.), Directrices del IPCC 2006 para los Inventarios Nacionales de Gases de Efecto Invernadero Volumen 4 Agricultura, 
Silvicultura y Otros Usos de la Tierra. (pp. 1-56). Japón: IGES.

IPCC. 2006d. Capítulo 3: Combustión Móvil. In S. Eggleston, L.; Buendia, K.; Miwa, T. Ngara \& K. Tanabe (Eds.), Directrices del IPCC de 2006 para los inventarios nacionales de gases de efecto invernadero Volumen 2. Energía (pp. 1-78). Japón: IGES.

Jancurová, M.; Minarovičová, L. \& Dandár, A. 2009. Quinoa - a Review. Czech Journal of Food Sciences, 27(2):71-79.

MAE. 2016. Primer Informe Bienal de Actualización del Ecuador a la Convención Marco de las Naciones Unidas sobre el Cambio Climático. Quito, Ecuador.

Mekonnen, M.M. \& Hoekstra, A.Y. 2010. Mitigating the water footprint of export cut flowers from the Lake Naivasha Basin, Kenya.

Mogollon, J.; Rivas, W.; Martinez, A.; Campos, Y., \& Marquez, E. 2015. Carbono orgánico del suelo en un gradiente altitudinal en la Península de Paraguaná, Venezuela, 15 .

Molden, D. \& de Fraiture, C. 2004. Investing in Water for Food, Ecosystems and Livelihoods. Blue Papel. In Comprehensive Assessment of water management in agriculture (p. 25). Stockholm, Sweden.

Monteros, A. 2016. Rendimientos de Quinua en el Ecuador 2016 (octubre 2015 - agosto 2016). Dirección de Análisis y Procesamiento de la Información Coordinación General del Sistema de Información Nacional Ministerio de Agricultura, Ganadería, Acuacultura y Pesca. Quito, Ecuador.

Pegasys Strategy and Development (PTY) LTD, WWF, Universidad Nacional Agraria La Molina UNALM, \& Autoridad Nacional del Agua - ANA. 2015. Huella hídrica del Perú. Sector agropecuario.

Pérez Arcos, S. isabel. 2012. Evaluación y análisis de la huella hídrica y el agua virtual de la producción agrícola en el Ecuador, 62.

PROINPA. 2011. La quinua: Cultivo milenario para contribuir a la seguridad alimentaria mundial. FAO. Bolivia.

Quiroga, C.; Escalera, R.; Aroni, G.; Bonifacio, A.; Gonzáles, J. A.; Villca, M.; Ruiz, A. 2014. Cap 3.1. Procesos Tradicionales e Innovaciones Tecnológicas en la Cosecha, Beneficiado e Industrialización de la Quinua. In Estado del arte de la quinua en el mundo en 2013 (pp. 258-296). FAO(Santiago de Chile) y CIRAD( Montpellier).

Ramiréz Cando, L. \& Spugnoli, P. 2016. A review of life cycle assessment: agroproducts modeling, 2010. (Icenv), 5-15. http://doi.org/10.17163/lgr.n24.2016.01

Rioja, A. 2002. Apuntes de Fitotecnia General E.U.I.T.A. Ciudad Real, España.

Rodríguez, R.; Garrido, A.; Llamas, M. \& Varela, C. 2004. La huella hidrológica de la agricultura española. Papeles de Agu Virtual, 2.
WRI \& WBCSD. 2011. Greenhouse Gas Protocol. Product Life Cycle Accounting and Reporting Standard. World Resources Institute, Washington DC, USA. Greenhouse Gas Protocol.

Zevallos, V.; Herencia, L. \& Ciclitira, P. 2014. CAPÍTULO: 3.6. Quinua, enfermedad celíaca y la dieta sin gluten. In Estado del arte de la quinua en el mundo en 2013 (pp. 358-374). FAO (Santiago de Chile) y CIRAD. 